\title{
A Compound Relay Protection Operation Criterion Based on Kirchhoff's Current Law and Abnormal Data Detecting Algorithm
}

\author{
Xuze Zhang1, Xiaoning Kang1, Hao Wang1, Qiyue Huang1, Yali Ma² \\ ${ }^{1}$ Shaanxi Key Laboratory of Smart Grid (Xi'an Jiaotong University), Xi'an Jiaotong University, Xi'an, China \\ ${ }^{2}$ Tianjin Power Transmission and Transformation Engineering Company, Tianjin, China \\ Email: dianqieefourier@163.com
}

How to cite this paper: Zhang, X.Z., Kang, X.N., Wang, H., Huang, Q.Y. and Ma, Y.L. (2017) A Compound Relay Protection Operation Criterion Based on Kirchhoffs Current Law and Abnormal Data Detecting Algorithm. Energy and Power Engineering, 9, 8894.

https://doi.org/10.4236/epe.2017.94B011

Received: January 16, 2017

Accepted: March 30, 2017

Published: April 6, 2017

\begin{abstract}
A compound relay protection operation criterion is proposed based on Kirchhoff's Current Law and abnormal data detecting algorithm. The abnormal data detecting algorithm are proposed after deep research on the abnormal data properties. The current transformer status monitoring system and current phase angle detecting system is introduced. A simulation model containing different power sources and loads is established in Matlab. The simulation results show that this compound criterion can work quickly and reliably in all conditions.
\end{abstract}

\section{Keywords}

Relay Protection, Abnormal Data, Smart Substation

\section{Introduction}

The abnormal data is one of the most serious problems threating the safety operation of smart substation. It is the data much bigger than the actual value and will influence the work of the relay protection system, make the protection start by error. There are many reports of the accident due to the influence of the abnormal data. How to decrease the influence of the abnormal data is a very urgent task.

After deeply studying the properties of the abnormal data and the characters of the smart substations, this paper proposes a new protection operation criterion by applying KCL (Kirchhoff's Current Law) and abnormal data detecting algorithm. The simulation result shows that this operation criterion works well. 


\section{Compound Criterion}

\subsection{Flow Chart of the Compound Criterion}

The flow chart of the compound criterion is shown in Figure 1. In the power system, no matter the system is working normally or in fault status, KCL is always satisfied. Besides the occurrence of abnormal data, there are two other conditions where KCL may not be satisfied:

1) When there is a fault on the busbar, KCL is not satisfied. The sum of the current phasors equals to the short-circuit current.

2) When the current transformer breaks down, KCL may not be satisfied.

In order to use KCL to detect the existence of abnormal data, it is necessary to overcome the influence of the two conditions above.

In the compound criterion, the status of current transformers and busbar protection status are firstly inspected. If the current transformers work well

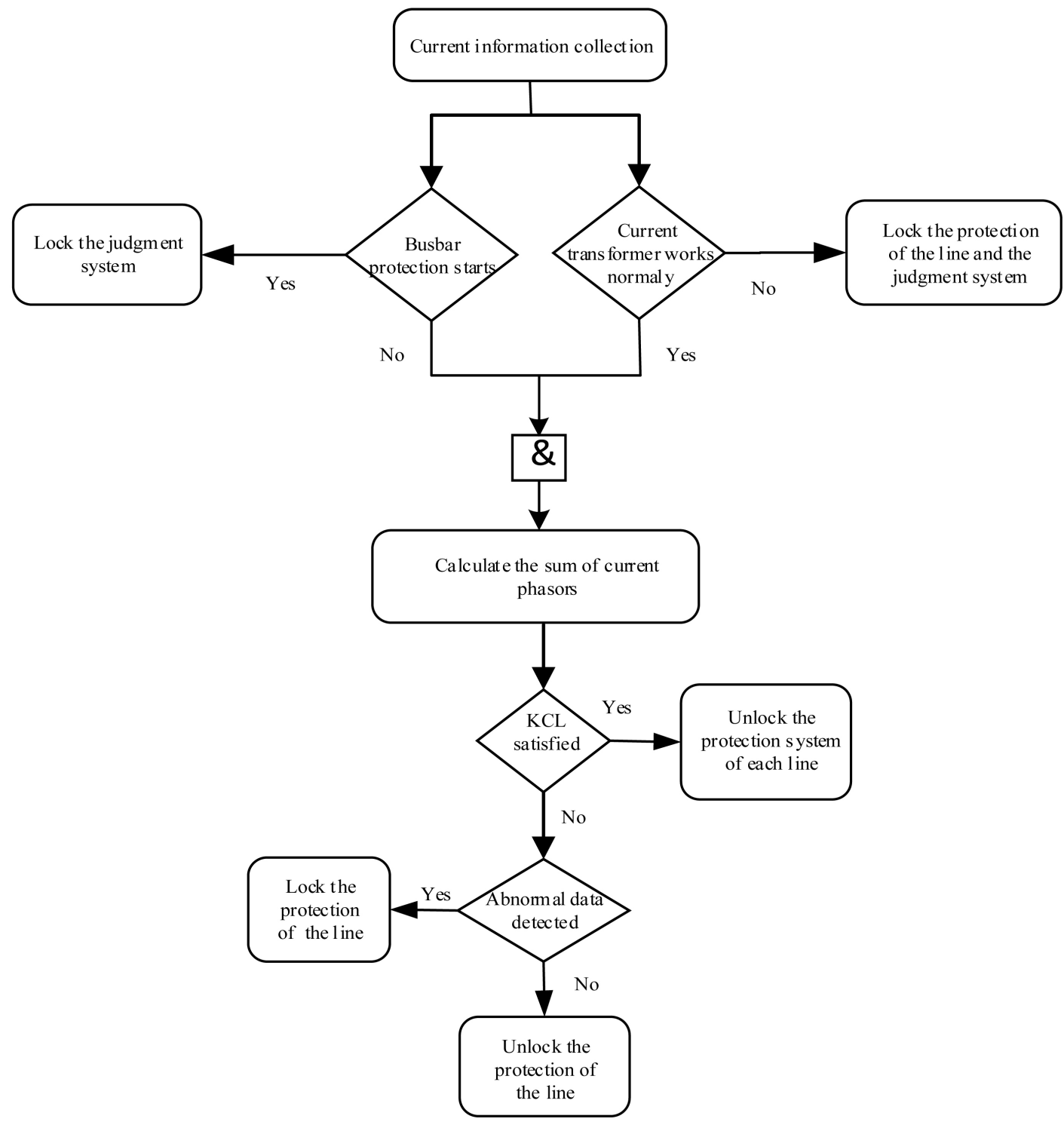

Figure 1. Flow chart of the compound criterion. 
and the busbar protection doesn't start, then the satisfaction of KCL is verified. If the sum of current phasors satisfies KCL, the system unlocks all the protection; if KCL isn't satisfied, the abnormal data detection algorithm is processed in each line. The system will lock the protection of the line detected with abnormal and unlock the protection of the line without abnormal data.

By this criterion, only the protection on the line with abnormal data is locked. The protection safety and reliability is improved. The abnormal data occurs in a relatively low frequency, so in most cases the judgment system only spends time on verifying KCL. The calculation time spent on verifying KCL is very short, so this criterion works much faster than the traditional criterions which only use abnormal data detecting algorithm, as the criterion in [1] [2] [3]. This is a very big advantage of this compound criterion.

\subsection{Abnormal Data Detecting Algorithm}

According to the power system theory, no matter the system is in normal operation or fault, the waveform of the current is always continuous. When an abnormal data occurs, there is a mutation on that point and the waveform is not continuous. Using this character, the abnormal data can be distinguished.

Supposing $f(\mathrm{x})$ is the sampled signal, the abnormal data occurs in the $n^{\text {th }}$ point.

$$
\begin{gathered}
\Delta f(n)=f(n)-f(n-1) \\
\Delta f(n+1)=f(n+1)-f(n)
\end{gathered}
$$

1) There is a mutation in the abnormal data, so we have:

$$
\left\{\begin{array}{l}
|\Delta f(n)|>\varepsilon_{1} \\
|\Delta f(n+1)|>\varepsilon_{1}
\end{array}\right.
$$

2) The waveform is not continuous at the abnormal point, so we have:

$$
|\Delta f(n)-\Delta f(n+1)|>\varepsilon_{2}
$$

$\varepsilon_{1}$ and $\varepsilon_{2}$ are two threshold values and could be calculated as following:

For a sinuous signal $f(t)=A \sin (\omega t+\phi)$,

$$
\begin{gathered}
f^{\prime}(t)=\omega A \cos (\omega t+\phi) \\
f^{\prime \prime}(t)=-\omega^{2} A \sin (\omega t+\phi)
\end{gathered}
$$

So $\left|f^{\prime}(t)\right| \leq \omega A$ and $\left|f^{\prime \prime}(t)\right| \leq \omega^{2} A$.We can choose $\varepsilon_{1}=K_{1} \omega A d t$, $\varepsilon_{2}=K_{2} \omega^{2} A d t . d t$ is the sample time interval, $K_{1}$ and $K_{2}$ are two safety factors.

$$
A=\sqrt{f^{2}(t)+\left(\frac{f^{\prime}(t)}{\omega}\right)^{2}}
$$

In order to increase the speed of the detection algorithm, for the $n^{\text {th }}$ point, we can use the average amplitude calculated by point $n-1, n-2$ and $n-3$ directly instead of calculating the amplitude at the $n^{\text {th }}$ point. 


\subsection{Current Transformer Status Real-Time Monitoring}

It is very important to know whether the CT (current transformer) is functioning normally in this algorithm. According to the real engineering experience, the CT status can be classified to short-circuit, open-circuit and normal operation and the status is related to the CT current loop impedance. Reference [4] proposes a CT status monitoring method, as shown in Figure 2.

The current loop consists of $\mathrm{CT}_{0}$ and $\mathrm{CT}_{1} \cdot \mathrm{CT}_{0}$ is a double-output current transformer. The status of $\mathrm{CT}_{0}$ can be obtained by measuring the impedance value of $\mathrm{CT}_{1}$. When $\mathrm{CT}_{0}$ is in short-circuiting, normal operation and open-circuit status, the impedance of $\mathrm{CT}_{1}$ changes much, which leads to the output voltage in the secondary side varies differently. The experimental results by [4] is shown in Table 1 .

From the experimental result, we can find that there is a big gap of the output voltage among the three statuses. By observing the output voltage, we can monitor the current transformer's real time status.

\subsection{Current Phase Real-Time Measurement}

In order to sum up the current phasor, it is necessary to measure the current phase. In this paper, the soft phase lock loop (Figure 3) is chosen to obtain the real-time phase as shown in [5].

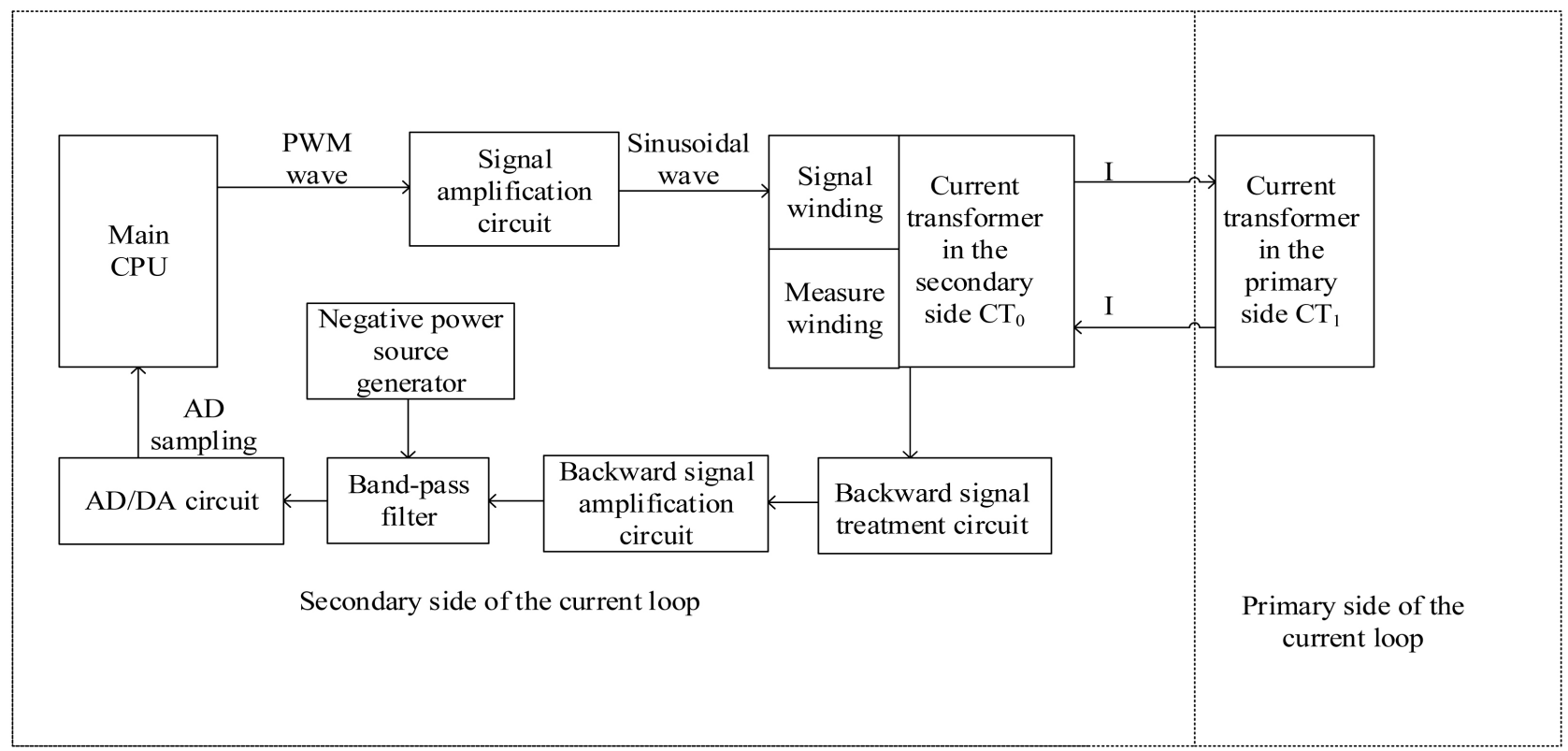

Figure 2. Structure of CT status monitoring facility.

Table 1. Output voltage in different status.

\begin{tabular}{cc}
\hline CT Status & Output voltage (V) \\
\hline Short-circuit & 0.2 \\
Normal operation & $0.4-1.4$ \\
Open-circuit & 1.8 \\
\hline
\end{tabular}




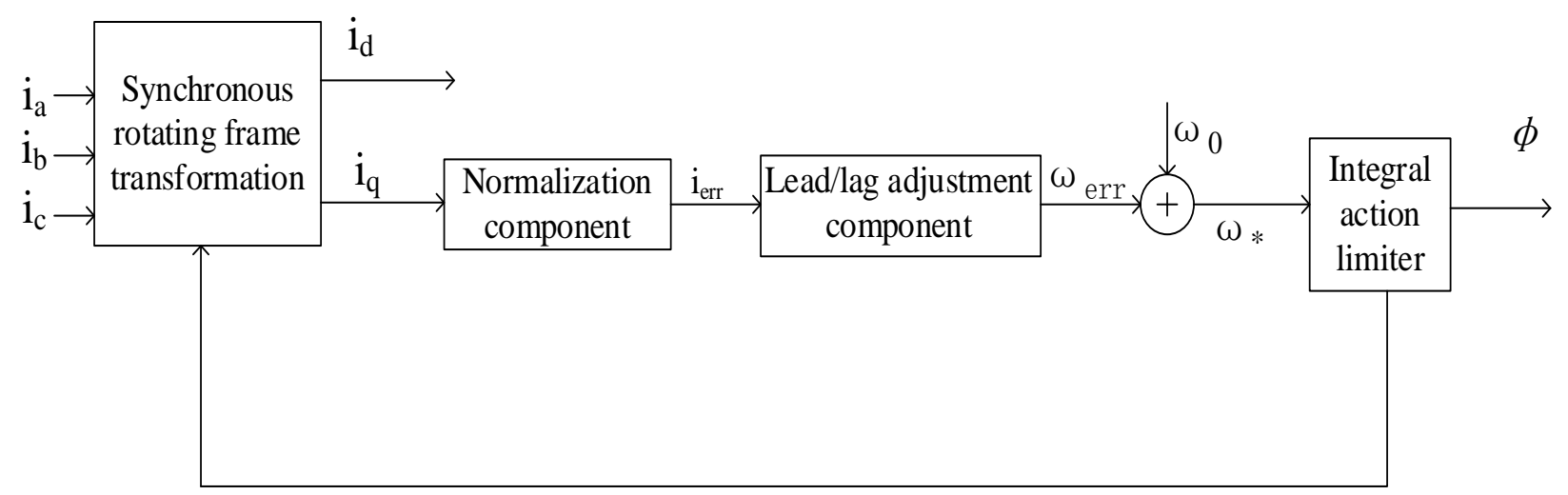

Figure 3. Frame of soft phase lock loop.

The three-phase current vector is,

$$
i=\left[\begin{array}{l}
i_{a} \\
i_{b} \\
i_{c}
\end{array}\right]=\left[\begin{array}{l}
I \cos (\omega t) \\
I \cos (\omega t-\theta) \\
I \cos (\omega t+\theta)
\end{array}\right]
$$

$I$ is the phase-to-ground amplitude of the current, $\theta=120^{\circ}$. The phase angle of phase $\mathrm{A}$ is $\phi=\omega t$, the estimated phase angle of phase $\mathrm{A}$ is $\phi^{\prime}=\phi-\Delta \phi, \Delta \phi$ is the error of the estimated phase.

After exhausting the synchronous reference frame transformation with $\phi^{\prime}$ chosen as the rotating phase, we can get

$$
\left[\begin{array}{c}
i_{d}(t) \\
i_{q}(t)
\end{array}\right]=\sqrt{\frac{2}{3}} I\left[\begin{array}{c}
\cos \left(\omega t-\phi^{\prime}\right) \\
\sin \left(\omega t-\phi^{\prime}\right)
\end{array}\right]=\sqrt{\frac{2}{3}} I\left[\begin{array}{c}
\cos (\Delta \phi) \\
\sin (\Delta \phi)
\end{array}\right]
$$

$i_{d}(t)$ and $i_{q}(t)$ are the components on axis $\mathrm{d}$ and axis $q$.

We can find from Equation (9): if there is no error in the estimated phase $\phi^{\prime}$, $\Delta \phi=0, i_{q}(\mathrm{t})=0$; if $\Delta \phi \neq 0$, the value of $\Delta \phi$ is related to the value of $i_{q}(\mathrm{t})$. After the lead/leg adjustment component, the error of estimated angular frequency is obtained. By the adjustment of angular frequency, the accurate phase and angular frequency of the current is obtained.

\section{Simulation}

In order to test the effectiveness of the criterion, a simulation model is established as shown in Figure 4.

In the simulation system, $G_{1}, G_{2}, G_{3}, G_{4}$ are four three-phase sources; $M_{1}, M_{2}$, $M_{3}$ and $M_{4}$ are four three-phase loads; $I$ is the bus bar of the system. The parameters of the four sources and four loads are chosen differently in order to test the effectiveness of the criterion in all possible conditions. The parameters of the sources and charges are shown in Table 2 and Table 3.

The simulation result of the protection of transmission line $\mathrm{L}_{1}$ is shown in $\mathrm{Ta}$ ble 4 .

Case 1: There are no abnormal data in the system.

Case 2: The abnormal data occurs only in $L_{1}$. 


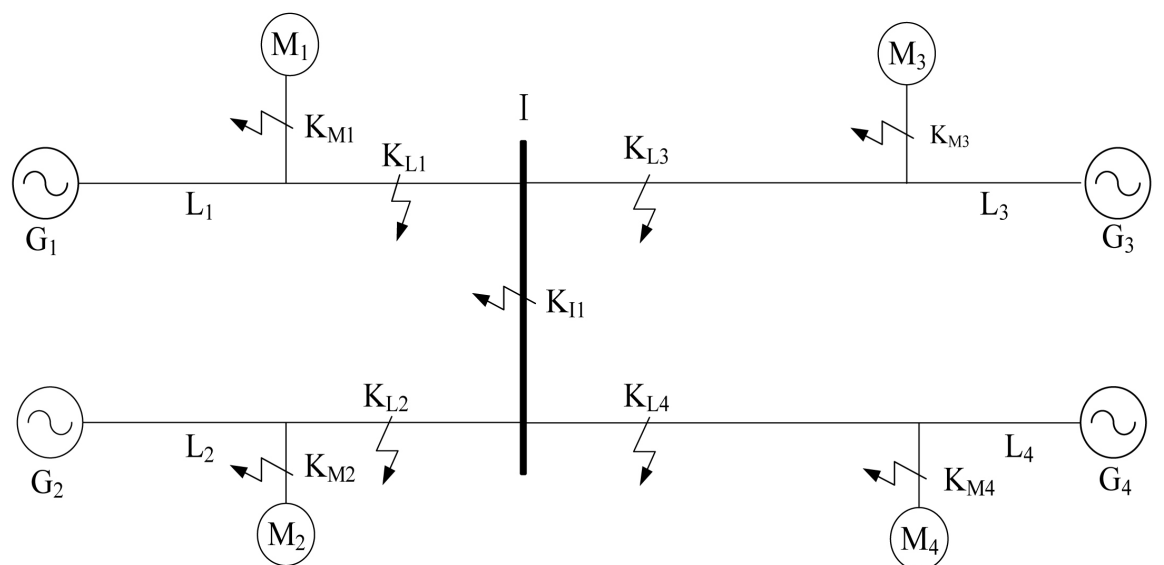

Figure 4. Simulation model.

Table 2. Source parameters.

\begin{tabular}{cccc}
\hline Source name & $\begin{array}{c}\text { Phase-to-phase rms } \\
\text { voltage }(\mathrm{kV})\end{array}$ & Power factor & Initial angle of phase $A\left(^{\circ}\right)$ \\
\hline$G_{1}$ & 35 & 0.8 & 0 \\
$G_{2}$ & 50 & 0.9 & 90 \\
$G_{3}$ & 50 & 0.9 & 180 \\
$G_{4}$ & 35 & 0.8 & 270 \\
\hline
\end{tabular}

Table 3. Load parameters.

\begin{tabular}{cccc}
\hline Load name & Active power $(\mathrm{kW})$ & Power factor & Load character \\
\hline M1 & 4000 & 0.9 & Inductive \\
M2 & 4000 & 0.9 & Capacitive \\
M3 & 8000 & 0.6 & Inductive \\
M4 & 8000 & 0.6 & Capacitive \\
\hline
\end{tabular}

Case 3: The abnormal data occurs in at least one line among $L_{2}, L_{3}$ and $L_{4}$.

Case 4: The abnormal data occurs in $L_{1}$ and at least one line among $L_{2}, L_{3}$ and $L_{4}$.

\section{Analysis of Simulation Results and Conclusion}

It can be concluded from the simulation results that regardless of the character of the sources and charges, by this operation criterion, the influence of abnormal data can always be avoided. This criterion can improve the reliability of the protection.

The electronic transformers are being more and more widely used, the research on how to detect the abnormal data and how to avoid the influence of abnormal data is becoming more and more important. How to make the protections keep working normally instead of unlocking them when the abnormal data 
Table 4. Simulation results.

\begin{tabular}{|c|c|c|c|}
\hline Fault position & $\begin{array}{l}\text { Abnormal } \\
\text { data }\end{array}$ & $\begin{array}{l}\text { Theoretical command on } \\
\text { the protection on } L_{1}\end{array}$ & $\begin{array}{l}\text { Real command on } \\
\text { the protection on } L_{1}\end{array}$ \\
\hline KI1 & $\begin{array}{c}\text { Case } 1,2, \\
3,4\end{array}$ & Unlock the protection & Unlock the protection \\
\hline KL1 & Case 1 & Unlock the protection & Unlock the protection \\
\hline KL1 & Case 2 & Lock the protection & Lock the protection \\
\hline KL1 & Case 3 & Unlock the protection & Unlock the protection \\
\hline KL1 & Case 4 & Lock the protection & Lock the protection \\
\hline KL2 or KL3 or KL4 & Case 1 & Unlock the protection & Unlock the protection \\
\hline KL2 or KL3 or KL4 & Case 2 & Lock the protection & Lock the protection \\
\hline KL2 or KL3 or KL4 & Case 3 & Unlock the protection & Unlock the protection \\
\hline KL2 or KL3 or KL4 & Case 4 & Lock the protection & Lock the protection \\
\hline KM1 & Case 1 & Unlock the protection & Unlock the protection \\
\hline KM1 & Case 2 & Lock the protection & Lock the protection \\
\hline KM1 & Case 3 & Unlock the protection & Unlock the protection \\
\hline KM1 & Case 4 & Lock the protection & Lock the protection \\
\hline $\mathrm{KM} 2$ or KM3 or KM4 & Case 1 & Unlock the protection & Unlock the protection \\
\hline $\mathrm{KM} 2$ or KM3 or KM4 & Case 2 & Lock the protection & Lock the protection \\
\hline $\mathrm{KM} 2$ or KM3 or KM4 & Case 3 & Unlock the protection & Unlock the protection \\
\hline KM 2 or KM 3 or KM4 & Case 4 & Lock the protection & Lock the protection \\
\hline
\end{tabular}

occurs should be the research direction in the future.

\section{References}

[1] Zhao, L., Qian,Y.H., Liu, H.J. and Li, Y.X. (2010) Abnormal Data Resisting Method in Digital Substation.Automation of Electric Power System,19, 97-99.

[2] Wu, W.J. and Zhang, J.W. (2015)Aflying Spot Detection Algorithm For Continuous Sampling Based on Removable Discontinuous Points. Power System Protection and Control, 4, 18-24.

[3] Wu,T.H., Jiang, L.H., Li, Y.J., Wu, H., Chen, J.P. and Deng, Y.P. (2011) Real Time Identification of Abnormal Sampling Data of Power System. Automation of Electric Power System, 23, 95-98.

[4] Xiao, Y. and Zhou, S.L. (2010) Design and Implementation Of Intelligent Detection Method for Secondary Side Circuit Fault of Current Transformer. Power System Protection and Control,12,115-120.

[5] Huang, J.M., Wu, C.H. and Xu, F.Q. (2013) Phase Detection of Fundamental Positive Sequence Voltage Based on Sequence-Decoupled Resonant Controller.Power System Technology,9, 667-672. 
Submit or recommend next manuscript to SCIRP and we will provide best service for you:

Accepting pre-submission inquiries through Email, Facebook, LinkedIn, Twitter, etc. A wide selection of journals (inclusive of 9 subjects, more than 200 journals)

Providing 24-hour high-quality service

User-friendly online submission system

Fair and swift peer-review system

Efficient typesetting and proofreading procedure

Display of the result of downloads and visits, as well as the number of cited articles Maximum dissemination of your research work

Submit your manuscript at: http://papersubmission.scirp.org/

Or contact epe@scirp.org 